Supplement of Atmos. Meas. Tech., 11, 3091-3109, 2018

https://doi.org/10.5194/amt-11-3091-2018-supplement

(c) Author(s) 2018. This work is distributed under

the Creative Commons Attribution 4.0 License.

(c) (i)

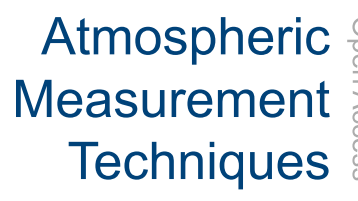

Supplement of

\title{
Evaluating tropospheric humidity from GPS radio occultation, radiosonde, and AIRS from high-resolution time series
}

Therese Rieckh et al.

Correspondence to: Therese Rieckh (rieckh@ucar.edu)

The copyright of individual parts of the supplement might differ from the CC BY 4.0 License. 


\section{immediate}

\section{Co-Location of ERA, RO, RS, and AIRS}

A map with the locations of the 4 chosen RS stations is shown in Fig. S1. Details about the co-location process are depicted in Fig. S2.

\section{${ }_{5} 22007$ annual mean profiles}

The constant value CLIMO (ERA 2007 annual mean) is used to normalize all data sets. The values for CLIMO are listed in Table S1.

3 Setting the stage: ERA 2007 time series of $N, T, q$,

10 and $\mathrm{RH}$

Time series for ERA refractivity, temperature, specific humidity, and relative humidity for 2007 are shown in Fig. S3 for Guam and in Fig. S4 for Ishigakijima. All data are interpolated to a common $25 \mathrm{hPa}$ grid.

\section{${ }_{15} 4$ Normalized difference for specific humidity between various data sets and ERA}

The Minamidaitojima $2007 \mathrm{RH}$ time series are shown in Fig. S5 (top). Time series for $q$ normalized differences between various data sets and ERA are shown in Fig. S5 (panels 20 2-5) and Fig. S6.

\section{Normalized difference for refractivity between various data sets and ERA}

Time series for refractivity $N$ normalized differences between various data sets and ERA are shown in Fig. S7 and ${ }_{25}$ Fig. S8. 


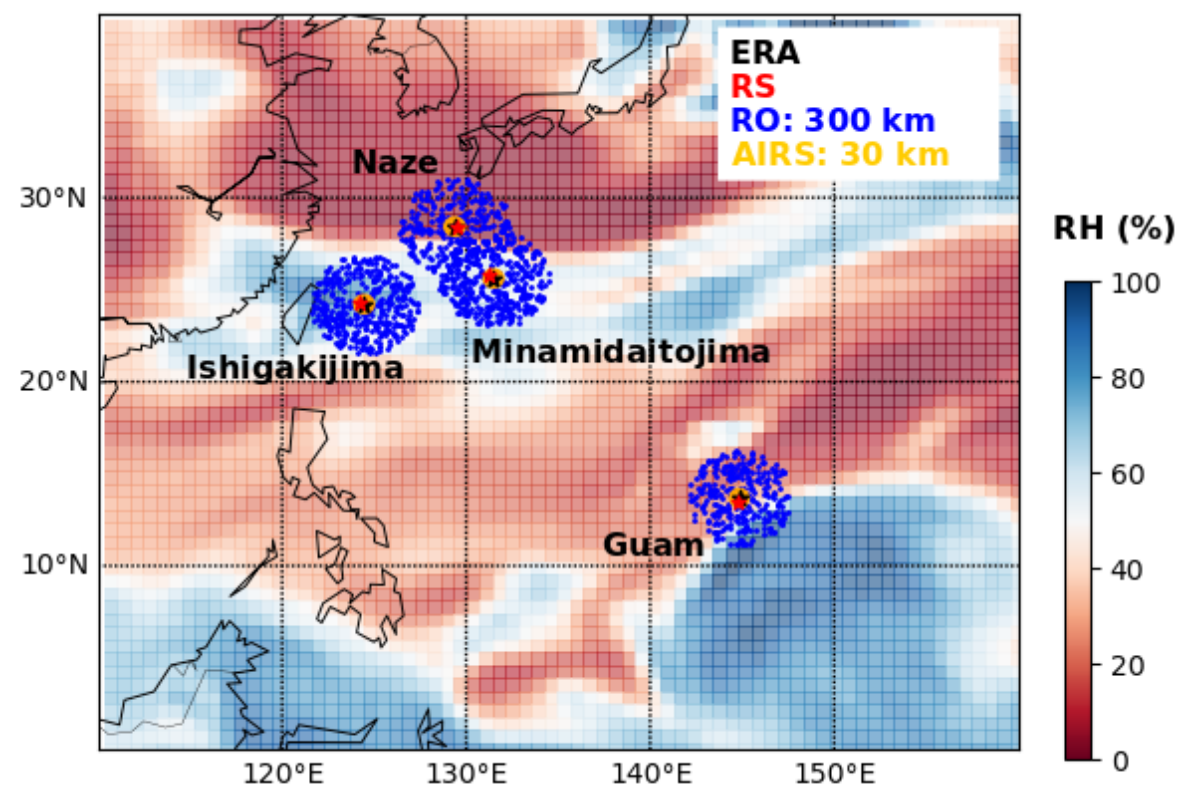

Figure S1. Map with the locations of Guam, Ishigakijima, Minamidaitojima, and Naze, and the locations of the data sets: ERA (black star), radiosonde (red star), RO (blue dots), and AIRS (yellow circle). The ERA relative humidity field at $700 \mathrm{hPa}$ for one day is shown in the background.

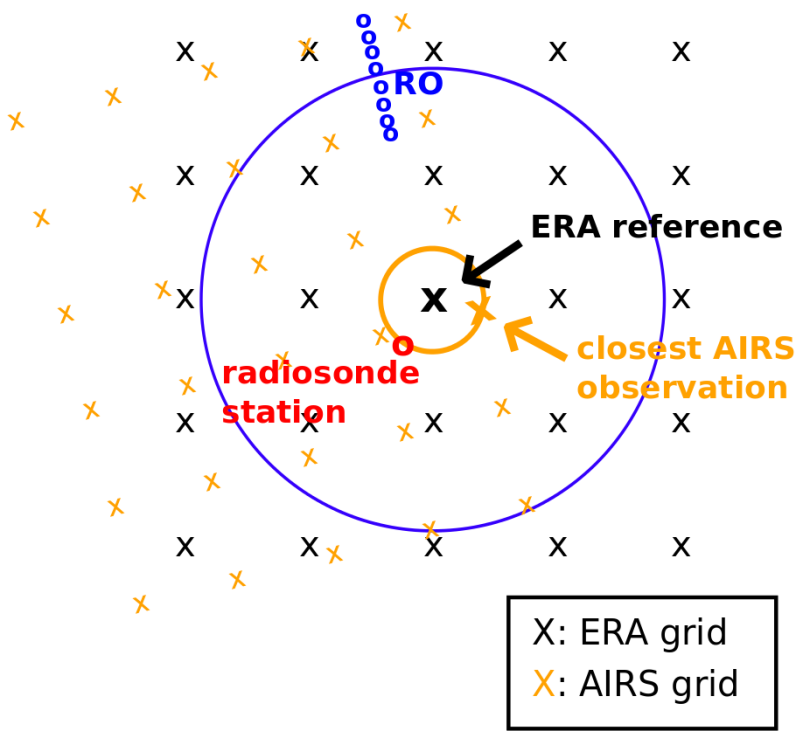

Figure S2. Sketch of the co-location of RO, RS, and AIRS with the ERA reference point for one point in time. ROs are chosen if their mean tangent point is within $300 \mathrm{~km}$ from the ERA reference. 
Table S1. Mean annual values for refractivity, specific humidity, relative humidity, and temperature for ERA in 2007, interpolated on a $25 \mathrm{hPa}$ grid.

\begin{tabular}{|c|c|c|c|c|c|c|c|c|c|c|c|c|c|c|c|c|}
\hline \multirow{2}{*}{$\begin{array}{r}\text { Pressure } \\
(\mathrm{hPa})\end{array}$} & \multicolumn{4}{|c|}{ Refractivity (N-units) } & \multicolumn{4}{|c|}{ Specific Humidity $\left(\mathrm{g} \mathrm{kg}^{-1}\right)$} & \multicolumn{4}{|c|}{ Relative Humidity (\%) } & \multicolumn{4}{|c|}{ Temperature $\left({ }^{\circ} \mathrm{C}\right)$} \\
\hline & Guam & Ishi & Mina & Naze & Guam & Ishi & Mina & Naze & Guam & Ishi & Mina & Naze & Guam & Ishi & Mina & Naze \\
\hline 300 & 96.7 & 96.8 & 97.0 & 97.5 & 0.3 & 0.3 & 0.3 & 0.3 & 41.6 & 37.8 & 38.8 & 40.3 & -29.8 & -30.3 & -30.9 & -32.1 \\
\hline 325 & 103.1 & 103.3 & 103.5 & 104.1 & 0.4 & 0.4 & 0.4 & 0.4 & 39.1 & 37.6 & 38.3 & 40.7 & -25.6 & -26.5 & -27.0 & -28.5 \\
\hline 350 & 109.8 & 110.3 & 110.4 & 111.2 & 0.6 & 0.6 & 0.5 & 0.5 & 36.6 & 37.5 & 37.7 & 41.2 & -21.5 & -22.6 & -23.2 & -24.9 \\
\hline 375 & 116.4 & 117.1 & 117.1 & 117.9 & 0.7 & 0.7 & 0.7 & 0.6 & 35.6 & 37.7 & 37.5 & 40.9 & -18.1 & -19.5 & -20.1 & -21.8 \\
\hline 400 & 123.5 & 124.3 & 124.3 & 125.1 & 0.9 & 0.9 & 0.9 & 0.8 & 34.5 & 37.9 & 37.3 & 40.6 & -14.8 & -16.4 & -16.9 & -18.7 \\
\hline 425 & 130.4 & 131.3 & 131.1 & 132.0 & 1.1 & 1.1 & 1.0 & 1.0 & 35.0 & 38.3 & 37.1 & 40.6 & -12.0 & -13.7 & -14.2 & -16.0 \\
\hline 450 & 137.7 & 138.7 & 138.4 & 139.3 & 1.4 & 1.4 & 1.3 & 1.2 & 35.5 & 38.7 & 37.0 & 40.6 & -9.3 & -11.0 & -11.5 & -13.3 \\
\hline 475 & 145.0 & 146.0 & 145.6 & 146.4 & 1.7 & 1.7 & 1.5 & 1.5 & 36.9 & 39.7 & 37.9 & 41.3 & -6.9 & -8.6 & -9.1 & -10.8 \\
\hline 500 & 152.8 & 153.7 & 153.2 & 154.0 & 2.0 & 2.0 & 1.8 & 1.7 & 38.3 & 40.8 & 38.9 & 42.0 & -4.4 & -6.3 & -6.6 & -8.4 \\
\hline 525 & 160.4 & 161.4 & 160.8 & 161.4 & 2.3 & 2.3 & 2.1 & 2.0 & 39.7 & 42.4 & 40.7 & 43.0 & -2.4 & -4.2 & -4.5 & -6.3 \\
\hline 550 & 168.4 & 169.5 & 168.9 & 169.3 & 2.7 & 2.7 & 2.5 & 2.3 & 41.1 & 44.0 & 42.4 & 43.9 & -0.3 & -2.1 & -2.4 & -4.2 \\
\hline 575 & 176.4 & 177.7 & 176.9 & 177.1 & 3.0 & 3.1 & 2.9 & 2.6 & 42.8 & 46.1 & 44.4 & 45.3 & 1.5 & -0.2 & -0.6 & -2.4 \\
\hline 600 & 184.7 & 186.3 & 185.4 & 185.4 & 3.5 & 3.6 & 3.3 & 3.0 & 44.6 & 48.2 & 46.3 & 46.8 & 3.4 & 1.7 & 1.3 & -0.6 \\
\hline 625 & 193.1 & 195.0 & 193.8 & 193.5 & 3.9 & 4.0 & 3.7 & 3.4 & 45.8 & 50.2 & 48.1 & 48.2 & 5.3 & 3.4 & 3.1 & 1.1 \\
\hline 650 & 202.0 & 204.1 & 202.7 & 202.0 & 4.5 & 4.6 & 4.2 & 3.8 & 47.1 & 52.3 & 49.9 & 49.6 & 7.3 & 5.1 & 4.8 & 2.8 \\
\hline 675 & 211.2 & 213.3 & 211.5 & 210.3 & 5.0 & 5.1 & 4.7 & 4.1 & 48.7 & 54.6 & 51.6 & 50.7 & 9.0 & 6.7 & 6.3 & 4.3 \\
\hline 700 & 220.8 & 222.9 & 220.8 & 219.0 & 5.7 & 5.7 & 5.2 & 4.6 & 50.4 & 56.9 & 53.3 & 51.8 & 10.7 & 8.2 & 7.8 & 5.8 \\
\hline 725 & 231.1 & 232.4 & 229.9 & 227.5 & 6.3 & 6.2 & 5.6 & 4.9 & 53.1 & 59.0 & 55.0 & 52.6 & 12.1 & 9.5 & 9.1 & 7.0 \\
\hline 750 & 241.8 & 242.3 & 239.5 & 236.3 & 7.1 & 6.8 & 6.2 & 5.3 & 55.9 & 61.1 & 56.6 & 53.5 & 13.6 & 10.8 & 10.3 & 8.3 \\
\hline 775 & 253.9 & 252.4 & 249.5 & 245.3 & 8.1 & 7.3 & 6.7 & 5.7 & 60.1 & 63.4 & 59.3 & 54.9 & 14.8 & 12.0 & 11.4 & 9.4 \\
\hline 800 & 267.1 & 263.0 & 260.3 & 254.9 & 9.2 & 8.0 & 7.4 & 6.2 & 65.4 & 66.3 & 63.0 & 56.9 & 15.9 & 13.1 & 12.4 & 10.5 \\
\hline 825 & 281.5 & 274.7 & 271.9 & 265.7 & 10.4 & 8.7 & 8.2 & 6.8 & 71.5 & 70.5 & 67.4 & 61.3 & 16.9 & 14.0 & 13.4 & 11.5 \\
\hline 850 & 295.9 & 287.0 & 283.8 & 277.7 & 11.6 & 9.6 & 8.9 & 7.6 & 76.6 & 74.7 & 71.5 & 66.9 & 17.9 & 15.1 & 14.3 & 12.4 \\
\hline 875 & 310.2 & 299.6 & 295.8 & 290.2 & 12.8 & 10.5 & 9.7 & 8.5 & 80.5 & 78.1 & 75.0 & 72.5 & 19.1 & 16.1 & 15.4 & 13.5 \\
\hline 900 & 324.2 & 312.5 & 308.4 & 303.3 & 13.9 & 11.4 & 10.6 & 9.5 & 83.2 & 81.1 & 78.3 & 77.6 & 20.4 & 17.3 & 16.5 & 14.6 \\
\hline 925 & 337.6 & 325.4 & 321.5 & 316.1 & 14.9 & 12.4 & 11.6 & 10.4 & 84.2 & 83.0 & 80.9 & 80.5 & 21.8 & 18.6 & 17.8 & 16.0 \\
\hline 950 & 352.0 & 338.2 & 334.5 & 328.2 & 16.0 & 13.3 & 12.5 & 11.2 & 86.6 & 83.7 & 82.5 & 81.1 & 22.9 & 20.0 & 19.1 & 17.4 \\
\hline 975 & 363.4 & 349.7 & 346.1 & 338.9 & 16.7 & 14.0 & 13.3 & 11.9 & 84.3 & 81.8 & 81.2 & 79.1 & 24.6 & 21.6 & 20.7 & 19.1 \\
\hline 1000 & 371.6 & 358.8 & 354.5 & 347.3 & 17.1 & 14.5 & 13.7 & 12.3 & 78.0 & 76.7 & 75.8 & 74.1 & 26.6 & 23.6 & 22.7 & 21.1 \\
\hline
\end{tabular}



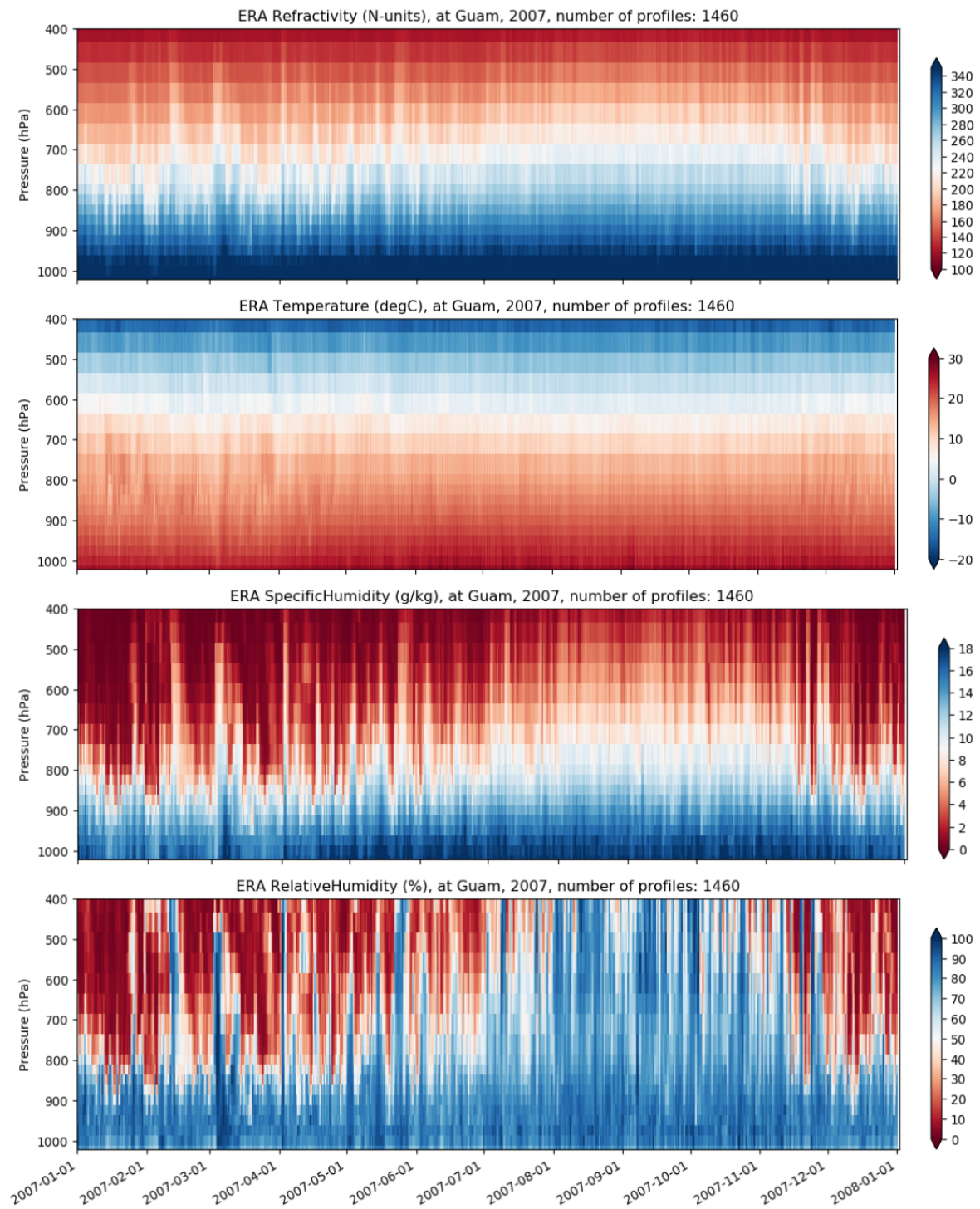

Figure S3. ERA 2007 time series at Guam for refractivity, temperature, specific humidity, and relative humidity (top to bottom). 

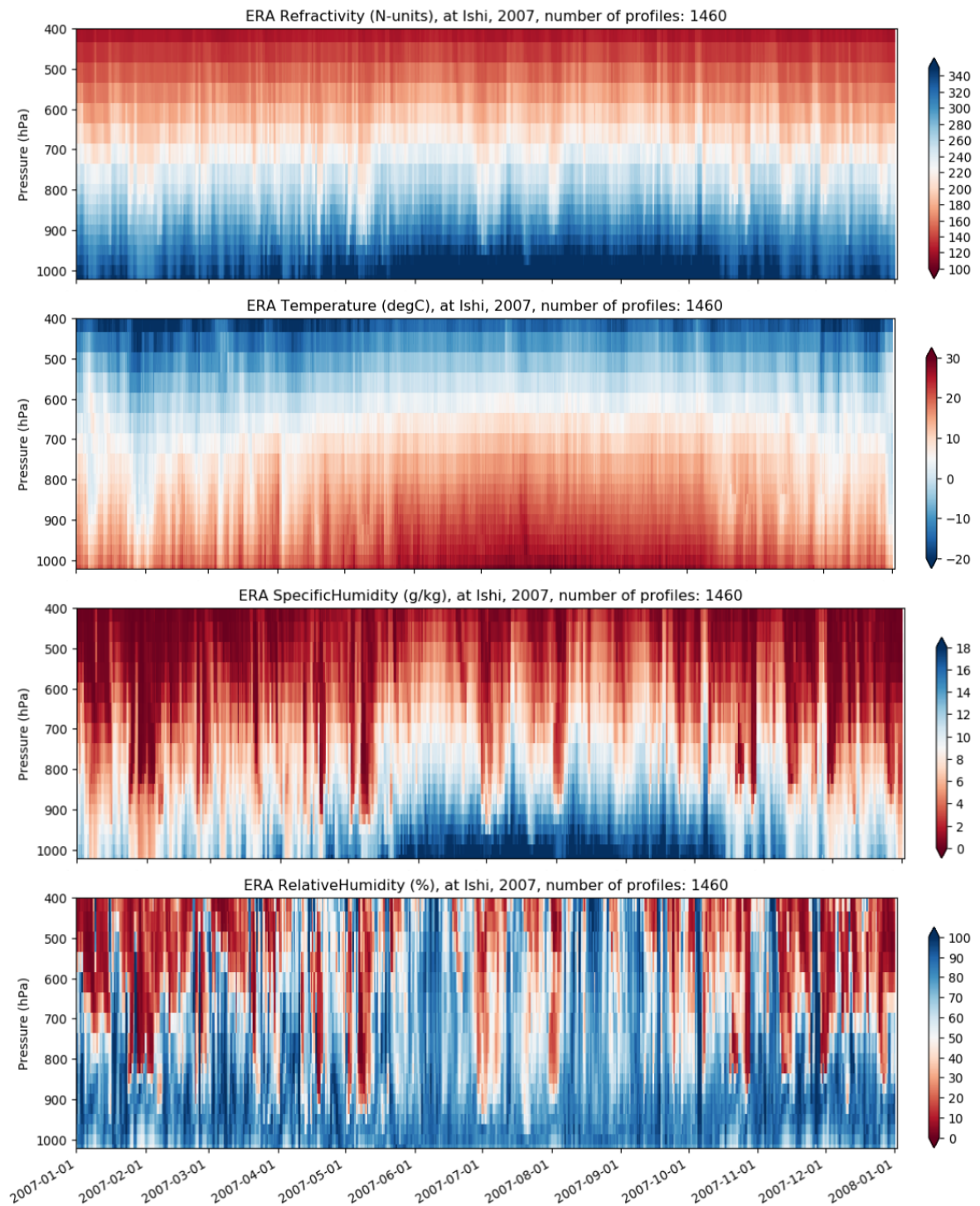

Figure S4. ERA 2007 time series at Ishigakijima for refractivity, temperature, specific humidity, and relative humidity (top to bottom). 


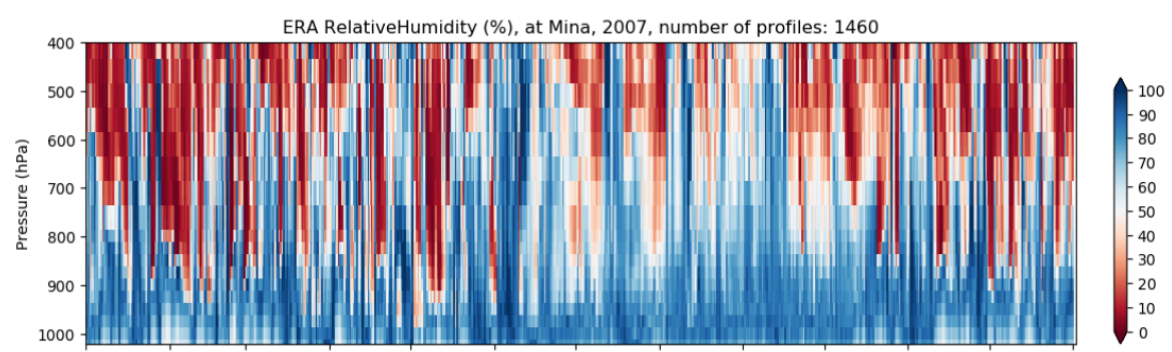

SpecificHumidity, PERSIST - ERA normalized difference (\%) at Mina, 2007, 1456 profiles

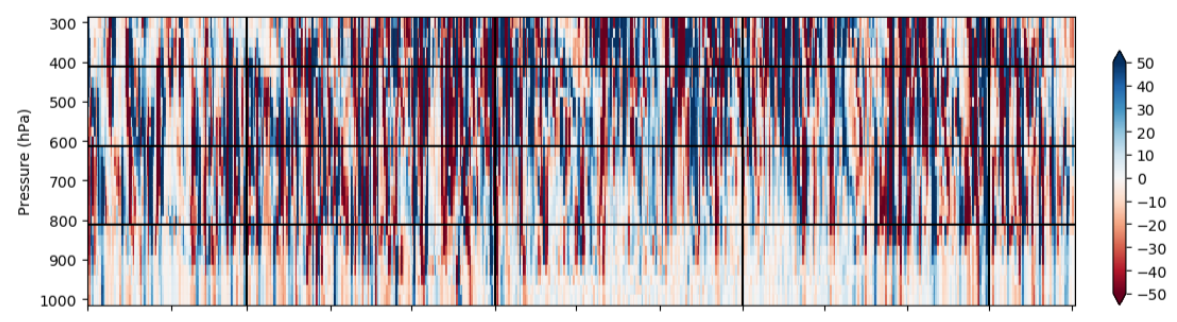

SpecificHumidity, GFS - ERA normalized difference (\%) at Mina, 2007, 375 profiles
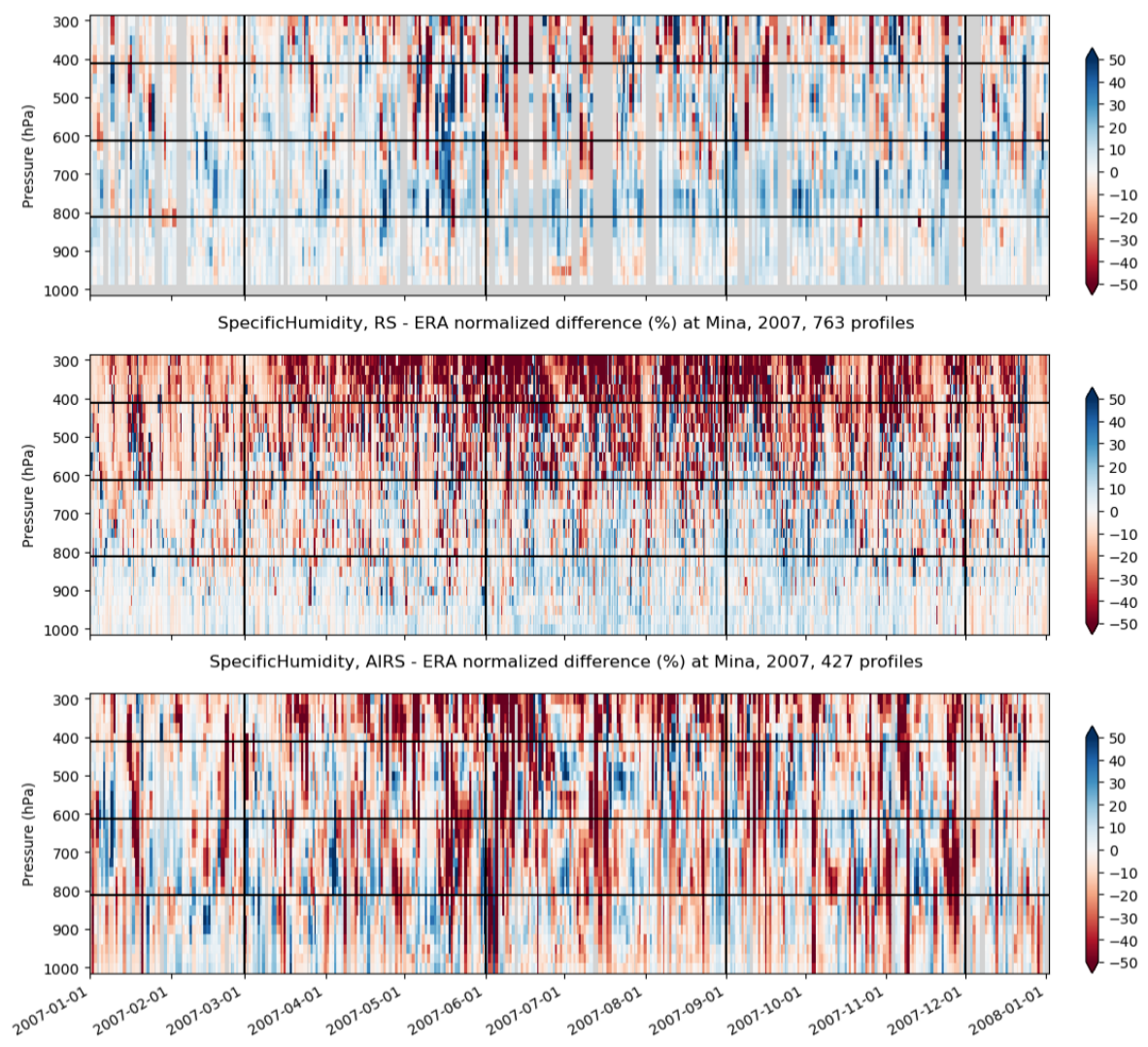

Figure S5. Top panel: 2007 time series of the relative humidity (\%) at Minamidaitojima over 2007 with blue representing moist air and red representing dry air. Other panels: Normalized difference of specific humidity for PERSIST, GFS, RS, and AIRS compared to ERA at Minamidaitojima, 2007. 
SpecificHumidity, UCAR direct - ERA normalized difference (\%) at Mina, 2007, 375 profiles
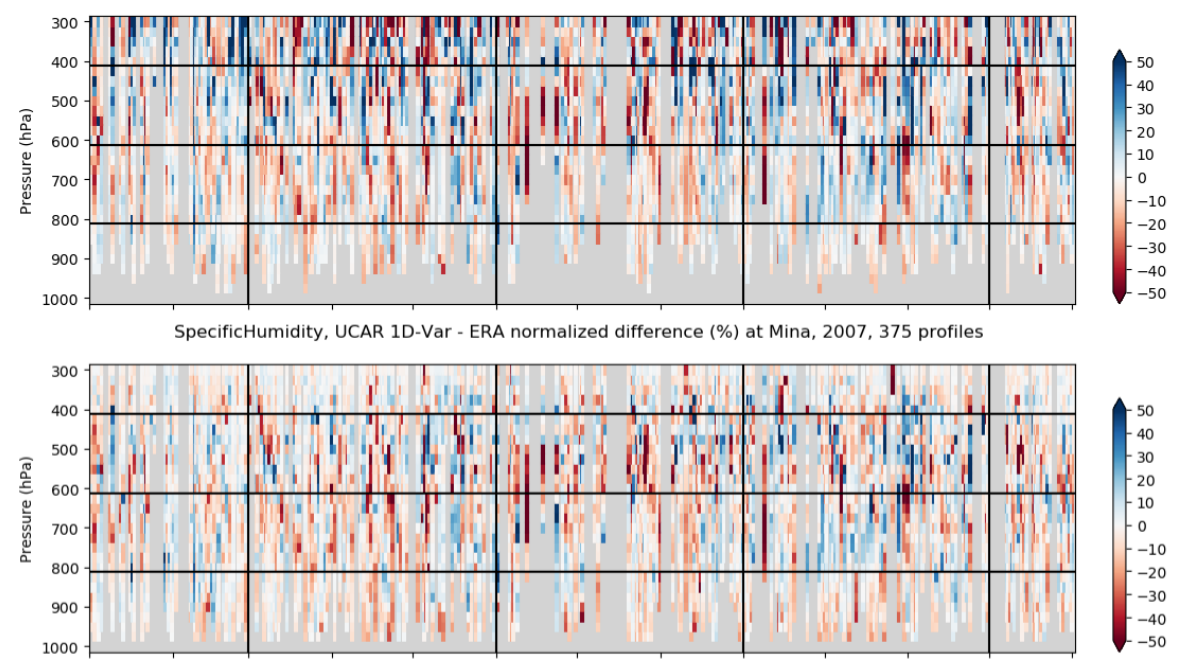

SpecificHumidity, WEGC 1D-Var - ERA normalized difference (\%) at Mina, 2007, 335 profiles

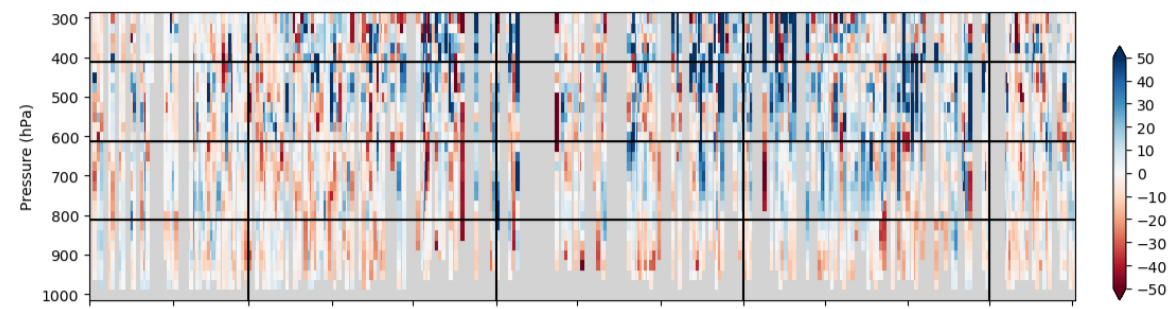

SpecificHumidity, JPL direct - ERA normalized difference (\%) at Mina, 2007, 223 profiles

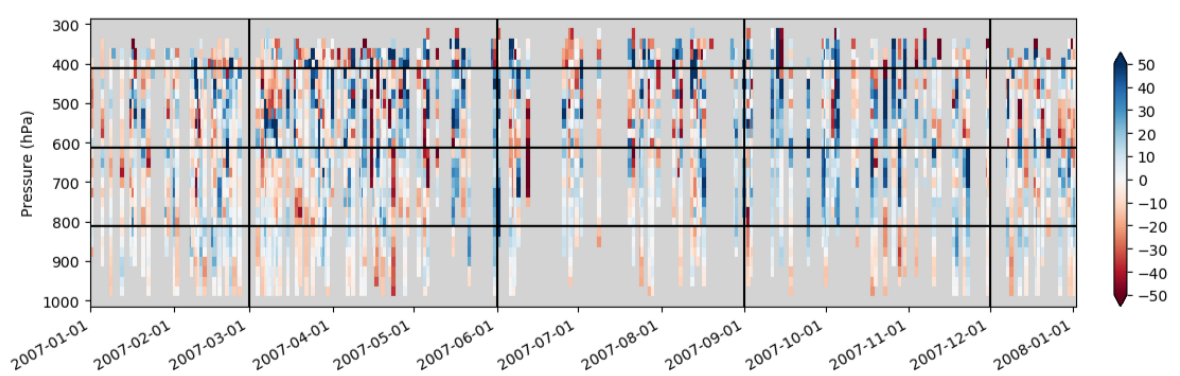

Figure S6. Normalized difference of specific humidity for the RO retrievals UCAR direct, UCAR 1D-Var, WEGC 1D-Var, and JPI direct compared to ERA at Minamidaitojima, 2007. 

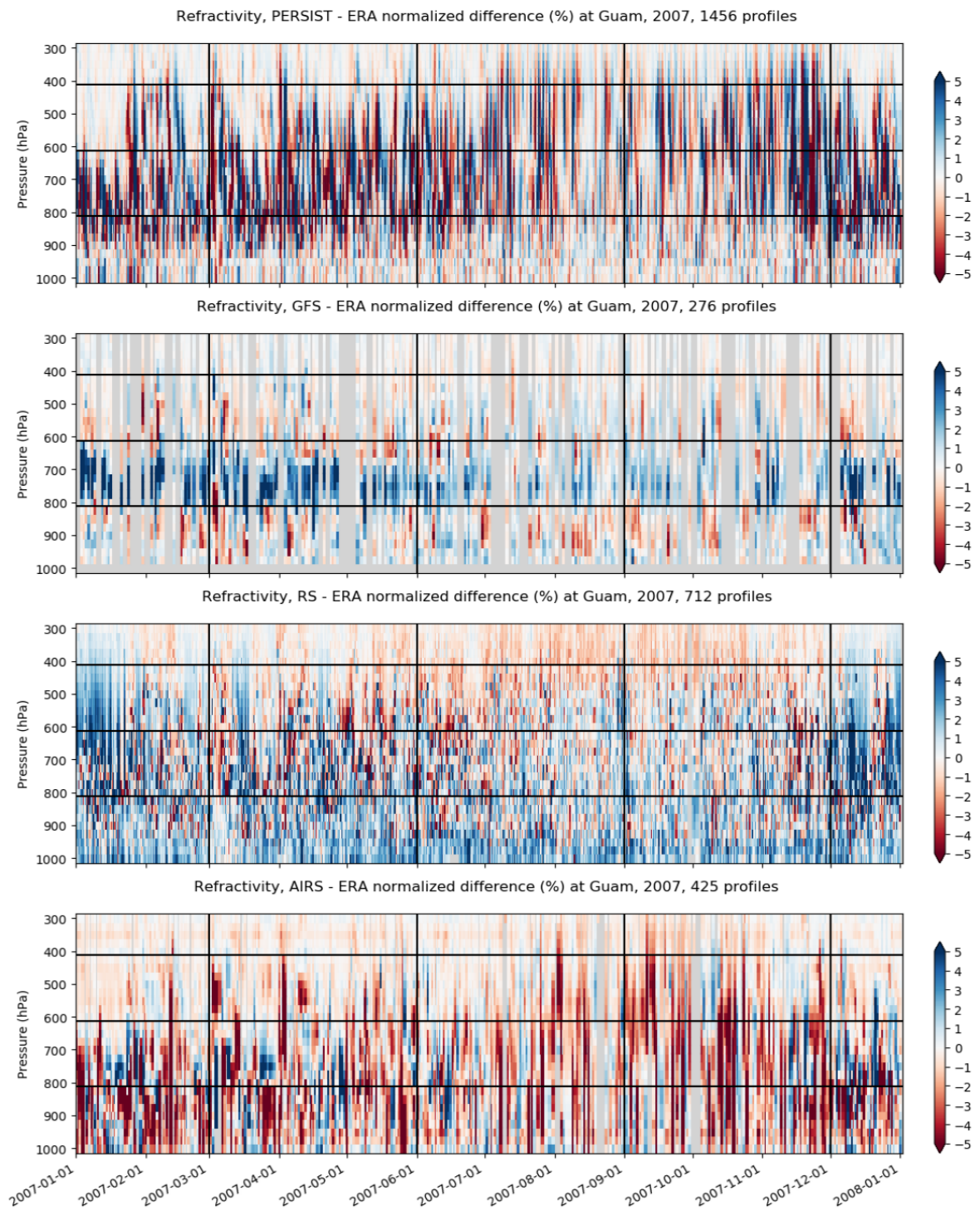

Figure S7. Normalized difference for refractivity for PERSIST, GFS, RS, and AIRS compared to ERA at Guam, 2007. 

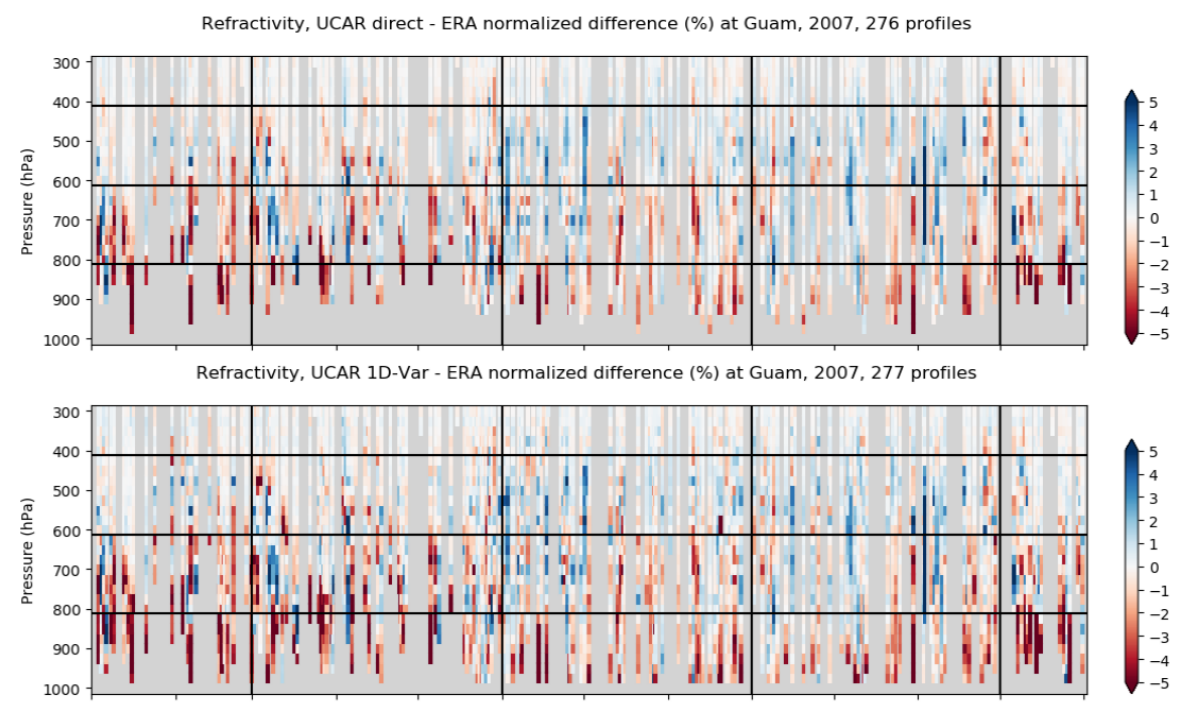

Refractivity, WEGC - ERA normalized difference (\%) at Guam, 2007, 239 profiles
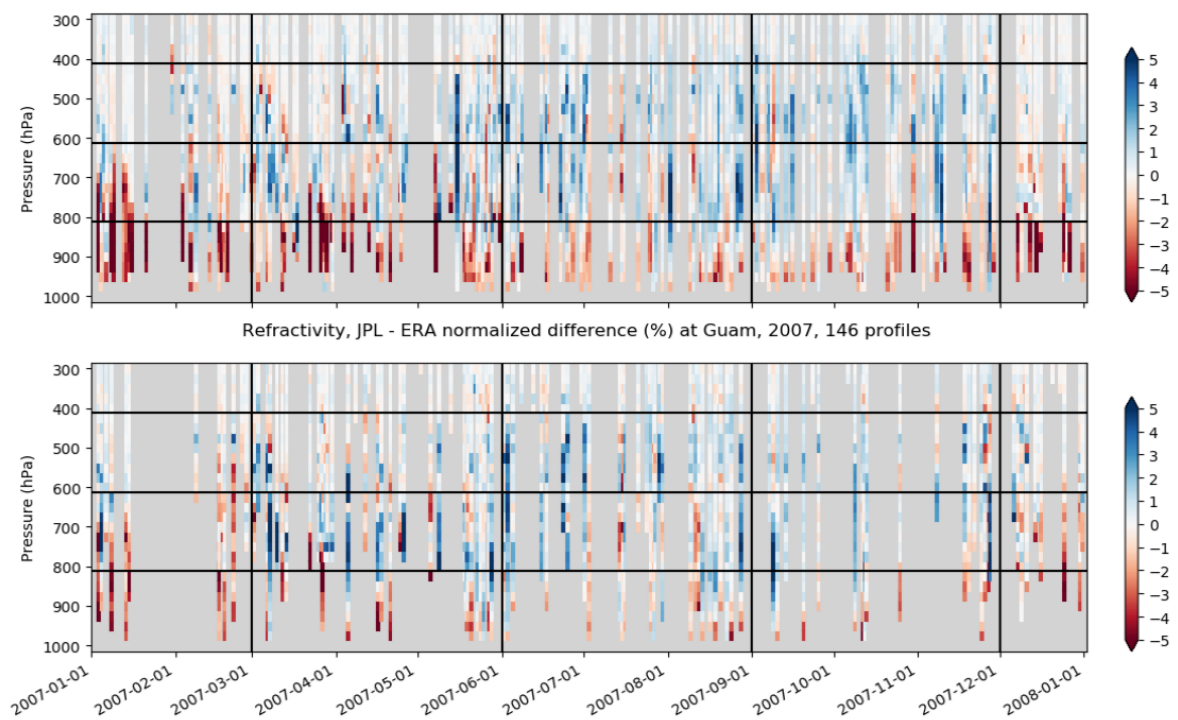

Figure S8. Normalized difference for refractivity for the RO retrievals UCAR direct, UCAR 1D-Var, WEGC 1D-Var, and JPI direct compared to ERA at Guam, 2007. 